\title{
Information Dissemination in Virtual Communities as Challenge to Real World Companies
}

\author{
Christopher Lueg \\ Department of Information Systems, University of Technology Sydney (UTS)
}

\begin{abstract}
The event of the Internet and its web-related services in particular has enabled business-related online communities (also referred to as communities of commerce) that are centered around companies and their web sites. Creating and nurturing such online communities is expected to be a key element in gaining and sustaining customer loyalty in the age of 'empowered fruit flies'. However, apart from such explicitly business-friendly communities, the Internet has also enabled a variety of business-independent online communities which may disseminate at the speed of light information about companies, their products, their bright sides, and their dark spots. The effectiveness of information sharing observed in a particular online community indicates that the Internet has enabled a variety of novel ways to affect companies, their reputations, and their businesses. A look at the relevant literature suggests that traditional security management is not yet prepared to cope with these new challenges.
\end{abstract}

\section{INTRODUCTION}

The importance of the Internet as communication medium has increased significantly over the past few years and so has commercial interest in online communities. The Internet and its web-related services in particular have enabled socalled communities of commerce (Bressler and Grantham, 2000) which are communities that are centered around companies and their web sites. Creating and nurturing such business-related online communities is expected to be a key element in gaining and sustaining customer loyalty in the age of 'empowered fruit flies' which denotes that "low-attention-span creatures with big wallets [...] move swiftly from one sweet fruit to the next in search of the best pricing, highest convenience, and quickest satisfaction" (Colony, 2000). Typical examples of communities of 
commerce are the social interactions that occur in the context of Amazon.com's virtual bookstore and eBay's virtual market place.

Apart from enabling such business-related online communities, the Internet has enabled a huge variety of online communities that are not related to businesses and that may pursue interests that are quite different from corporate interests. Such communities may be beneficial to a company they like by conveying a positive image but they may also be detrimental to a company's reputation and business by spreading information that contributes to a negative (or more realistic) image. Business-independent communities are difficult to address for companies and ways to influence or even control such communities are limited. It is tempting to underestimate the power of online communities-and online communication in general-as online communities may be relatively small in terms of members. Nevertheless, such communities may strongly effect a company. The lever is the virtually unlimited dissemination of information.

In this paper, we describe an online community that has formed to share experiences with fast food products and we outline that this community is an environment where effective information sharing is happening. Considering the growing importance of online communities and online communication in general, we argue that the Internet has enabled a variety of novel ways to affect companies, their reputations, and their businesses. A look at the relevant literature suggests that information management and security management are not yet prepared to cope with these new challenges. Companies in particular have to understand that the forces of the Internet can hardly be controlled; the challenge is to understand how to react to them (Bressler and Grantham, 2000).

\section{MOTIVATION: INFORMATION DISSEMINATION IN A COMMUNITY}

According to Williams and Cothrel (2000), online communities are groups of people who engage in many-to-many interactions online and form wherever people with common interests are able to interact. In this sense, the term "community" is used in a more general sense than in sociological research (e.g., Wellman and Gulia, 1999) where sharing beliefs or a feeling of belonging to the same community is considered essential for viewing a social groups as community.

In this paper, we focus on particular newsgroup which is situated in the global conferencing system Usenet news. Usenet being founded in the early Eighties is one of the oldest and probably the largest conferencing systems of its kind. Usenet is purely text-based and it is used by thousands of users who contribute more than a million messages per day, generating a daily network traffic of more than a hundred gigabytes. Collecting precise usage data is rather difficult as the largest part of user interaction with Usenet is invisible; messages only reflect active contributions to 
Usenet whereas the much more frequent activity of reading messages remains invisible (Lueg, 2000).

Research has found Usenet newsgroups be capable of forming and hosting communities (e.g., Roberts, 1998). Interest in Usenet is increasing (again) as newsgroups have been identified as places where is effective community building and information dissemination can be observed.

\subsection{The Online Community dafff}

In this paper, we focus on the Usenet newsgroup dafff. The name of the newsgroup is an abbreviation of the newsgroup's location within a particular internationally distributed newsgroup hierarchy. dafff qualifies as a community in the sense of Williams and Cothrel (2000) as its members are engaging in many-tomany interactions and the motivation for the engagement is the shared interest in certain products. In addition, dafff members share certain attitudes, such as expecting posters to behave according to the Usenet netiquette and its rules of good conduct. Also, they maintain shared artefacts, such as a list of answers to frequently asked questions (FAQ) and a web site that is used as a central repository for information, such as the FAQ, that are relevant to the newsgroup and their interests. The newsgroup's participants are well connected in terms of electronic communication so that information can be distributed rapidly. The shared interest in a particular topic is the basis for the newsgroup which suggests to view the newsgroup as a community of interest (Carotenuto et al., 1999).

The dafff newsgroup has formed to discuss "fast food" which is a generic name for food as offered by companies such as McDonald's, Burger King, KFC, Pizza Hut, Taco Bell, Hungry Jack's, Wendy's, and Subway. Fast food could be characterized as food that is prepared and consumed within minutes. Typical fast food products are hamburgers, French fries, pizzas, and sandwiches, which are usually served in combination with soft drinks, such as Coca Cola and Pepsi Cola.

\subsection{Information Sharing in the dafff Community}

Experiences with fast food and the respective restaurants are shared and discussed in the dafff newsgroup. Information being contributed by newsgroup members range from the quality of food and service (e.g., food temperature and consistency; speed of service; friendliness of employees; responsiveness to questions and critiques) in particular restaurants to regional differences in what restaurants offer. In one particular case, a newsgroup member reported that he or she observed the (in Germany strictly forbidden) re-use of food products that were returned by an unsatisfied customer.

Of particular interest are hamburger restaurants. In the case of one particular hamburger giant, information range from knowing which restaurants are operated by 
the giant itself and which are operated by franchise partners to details of internal quality control systems that are intended to ensure product quality. These quality control systems are a frequent topic as they directly influence the food experience in fast food restaurants. Fast food companies seem to face the problem that their freshly made products should be consumed within short periods of time to ensure product quality (depending on the actual product, products may be stored in the warmer for a few minutes). According to internal quality standards, products not served after this time should be discarded.

One of the two most popular hamburger companies marks its freshly made products according to the time left until the products should be discarded. Its main competitor uses a number-based flag system to indicate until when products may be kept. In both cases, details of the quality control systems and the meaning of the indicators used are not communicated to customers. As a consequence, customers ultimately have to trust the companies that only freshly made food is served and that overdue food is indeed discarded.

Some members of the newsgroup community, however, knew about the quality control systems used and contributed these information to discussions in the newsgroup. Almost all members of the newsgroup are now able to assess how fresh products really are when they are served. Observations reported by newsgroup members indicate that it happens that overdue food products are not discarded as demanded by internal company policies. In the case of the company using the flagbased system, it seems to happen that sometimes quality control indicators are manipulated to pretend a later production time. As a result, food served may not be as fresh as demanded by company policies. In the case of some restaurants of that particular company, newsgroup members reported they could observe that the flag system was not used at all so that it were impossible even for employees to assess freshness of products they were serving to customers.

information about the quality control system system, how it is intended to be used and how it may be abused, has become part of the community's "organizational memory" and is described in the newsgroup's FAQ. In this sense, it is not only information sharing but knowledge sharing that can be observed in the community as statements indicate that members effectively learned how to apply the information when visiting fast food restaurants.

\subsection{The Scope of the dafff Community}

The newsgroup's FAQ is available on the web site that is maintained by some of the newsgroup members. New participants in the newsgroup are pointed towards to web site when information related to topics covered by the FAQ are being requested. Discussions as well as the FAQ can easily be found when using a regular search engine, such as Google (http://www.google.com). In addition, it is reasonable to assume that members share their knowledge with real world friends and colleagues 
when physically visiting fast food restaurants. dafff members are physically located Germany and a few other countries which means that they are able to collect information from a rather large physical area.

Put in a nutshell, the scope of the community exceeds the particular newsgroup by far; the community and the corresponding web site have the potential to become a widely recognized fast food information resource on the Internet. Given the growing recognition of the Internet's importance to companies (e.g., Kalish, 1997; Reuters, 1997), it is not unlikely that some of the fast food companies discussed in the newsgroup are already monitoring the ongoing discussions. In fact, some members are known to be current employees of fast food companies discussed in the newsgroup.

Community members may also use other online communication channels, such as email, mailing lists, chats, instant messenger, and other newsgroups, to disseminate information they received in a community. Furthermore, members of online communities are real persons in the end who meet family members at home, friends at the movies, and colleagues at their workplaces which means that rumors can be expected to be disseminated in the real world as well.

Related to the problem of virtually unlimited information dissemination is that that communication is no longer limited to their specific social contexts. Search engines, such as Alta Vista and Google, preserve information for years and have empowered casual users to find information that was published almost anywhere on the Internet. Online archives, such as Deja.com (now Google.com) allow users to search billions of web pages and articles that were published in the global conferencing system Usenet news. This means that even casual users have access to a vast amount of information (positive and negative; true and false) that has been said about particular companies and their products. A further implication is that communication is removed from its original context. As Grudin (2001) puts it: "[...] capturing context digitally alters it fundamentally. The context that is captured is removed from its context, namely the context that is not captured". This de-situating is especially relevant when considering the community example discussed in this paper. A quick glance at the discussions in this community could suggest that "fast food bashing" occurs but in fact the community is a "fan" community mostly consisting of members that love fast food.

\subsection{Communities as Chance for Corporations}

From a business perspective, the dafff community is especially interesting as most community members share a positive attitude towards fast food despite some negative experiences. This means that the community and its web site are different from increasingly popular "revenge" web sites, such as McSpotlight (http://www.mcspotlight.org) or Living Wages (http://www.nikewages.com), 
providing information about the fast food giant McDonald's and the sports equipment giant Nike, respectively.

Empirical evidence exists that members of the dafff community are interested in increasing the quality of the products they like and the service they enjoy. Apart from revealing and discussing dark spots, newsgroup members also report on good experiences in particular restaurants and how they liked certain products (especially in the case of new and limited "special offers"). However, implications of the "quality problem" discussed above should not be underestimated. Discussion statements indicate that several newsgroup members understand the quality problem in such a way that they question the company's attitude towards its own quality standards in general which means that they question other standards of the company as well.

In the case of online communities with a rather positive attitude towards companies and their products, companies may have the chance to establish communication with online communities in order to prevent damage and to actually improve their services. For example, the observations concerning disregard of the internal quality control system could be used to investigate incidents.

\subsection{Supporting Communication With Online Communities}

The members of the dafff community are mainly located in Germany. A review of the German web sites of the two most popular fast food companies in the newsgroup suggests that additional communication channels would be required to establish communication between companies and online communities As reported in Lueg (2001a), the current web sites typically lack ways to provide feedback online or to communicate with each other. Instead, online customers are referred to sending faxes or providing feedback in restaurants. According to experiences discussed in the newsgroup, the latter can be at least frustrating if customers address disregard of the internal quality control systems. Reviews of the companies' web sites in countries all over the world showed similar results in most cases. Interestingly, Japan with its specific culture is treated differently.

A range of ways to support communication with business-friendly online communities has been outlined in Lueg (2001a). In a nutshell, the communication strategy should be based on interaction and feedback; the communication goal should be to prevent deterioration of reputation through isolated events and to improve reputation through transparency. The message should be that despite bad experiences the company still is a trustworthy company offering quality products, that the company does not intend to hide grievances, and that the company investigates and resolves incidents as soon as possible after they have been reported.

Certainly, providing additional communication channels involves certain risks. Appropriate communication requires considerable expertise (domain knowledge, 
legal knowledge, PR knowledge, etc.) and still communication may fail if the company's reaction to incidents does not meet what is expected.

\section{ONLINE COMMUNICATION AS CHALLENGE TO COMPANIES}

Providing new communication channels always introduces additional risks as communication channels may be abused for information-level Denial-of-Service (DoS) attacks (see below for a definition). In a more general sense, the speed with which information can be disseminated within online communities and among user of the Internet in general indicates that information dissemination in the age of the Internet should be considered a serious challenge for companies.

It is widely acknowledged that computer security is an important topic and the state-of-the-art in computer security provides some protection against threats ranging from hackers trying to break into corporate computer systems to DoS attacks. Companies should be able to reduce vulnerabilities as well as the potential impact of still successful attacks.

However, information sharing in Usenet newsgroups suggests that potentially threatening activities that are based on the virtually unrestricted dissemination of information are becoming more and more important. Examples for information that may be disseminated are rumors, gossip, urban legends, and last but not least purposely false information. Computer viruses, such as the Melissa virus or the more recent Love Letter virus, infecting computers all over the world within a few hours, have demonstrated the speed of information dissemination in the age of the Internet.

A famous example for the threat potential of information dissemination in Usenet newsgroups and on the Internet in general is the urban legend that the American designer Tommy Hilfiger appeared on the Oprah Winfrey Show (an extremely popular show in the US) and made racist comments about several groups, after which he was tossed off the set by Winfrey. In fact, Hilfiger has never appeared on or taped an episode of Winfrey's show but the legend spread so rapidly and generated so much controversy among customers and potential customers that the company was forced to respond on the net (Ulfelder, 1997). See Lueg (2001b) for a discussion of how online communication may affect real world companies.

It is necessary to distinguish such information-based attacks from network-based attacks, such as regular DoS attacks, as network-based attacks are easier to address due to their technical nature. Lueg (2001b) defines information-level attacks as attacks that are based on the dissemination of information in such a way that companies, their operations, and their reputations may be affected. Dissemination may be active as in the case of sending email or passive as in the case of setting up web sites. 
The primary lever of an information-level attack is the content of a message rather than its form. Sending faked inquiries to service accounts (e.g., feedback channels for online communities) to eat up resources would qualify as informationbased attack as it is the content of the messages that would provide the lever for the attack. Examples for information-based threats are the setting up of so-called revenge web sites and the disseminating of false or biased information as in the case of the false Hilfiger accusation. Dissemination of information that is likely to trigger specific counter reactions as in the case of "joe jobs" also qualifies as informationbased threat. A joe job denotes abusing an opponent's identity for spamming. The intended effect of a joe job is that the apparent sender is blamed for spamming (i.e., damaged reputation) with the ultimate goal that the opponent is being dropped by his Internet service provider.

The computer security related literature does not provide much information on how to address information-level threats. A look at the relevant literature suggests that the focus is on making corporate computer systems and networks secure in order to protect them against direct undesirable activities. Topics discussed at specialized computer security conferences, such as the Australasian Conference on Information Security and Privacy (ACISP), range from authentication and encryption to access control and intrusion detection. Threatening activities that are based on the dissemination of information, however, are happening outside secure environments. (Lueg, 2001b).

Companies, such as CyberAlert (http://www.cyberalert.com), IntelliSeek (http://www.intelliseek.com), and eWatch (http://www.ewatch.com), have recognized the threat potential and offer specific tools that allow to search the web and other information sources to find out "what is 'being said about [a] company and its products', and that provides 'a way to identify potentially damaging rumors' " (Manktelow, 2001). However, the strengths and weaknesses of these tools are largely unknown and information on how to incorporate information-level threats into security management are hard to find. See Lueg (2001b) for a discussion of the current situation. Ebbinghouse (2001) provides an overview of several ways to address problems with online information.

\section{CONCLUSIONS AND FUTURE RESEARCH DIRECTIONS}

In this paper, we have outlined that not only the creation of new business-related online communities (so-called communities of commerce) but also the appropriate consideration of existing online communities is increasingly important in the age of the Internet. As a matter of fact, companies cannot control information dissemination in online communities but they can (and they will have to) react to the fact that more and more of their real world customers are members in online 
communities as well. Moreover, the virtually unrestricted information dissemination on the Internet should be considered a serious challenge to companies.

The focus of this paper was on one particular community and its potential impact on real world companies. We are extending our investigation to cover communities in a variety of online environments as we consider the community under investigation as a representative for a new breed of online communities that can either support or harm companies.

Another strand of work focuses on the development of a framework for assessing information-level activities and the incorporation of information-level security management into existing security management frameworks.

\section{ACKNOWLEDGEMENTS}

The author would like to thank Ingrid Slembek and the anonymous reviewers for their comments on drafts of this paper, Robert James Steele for pointing to the Manktelow article, and Paul Verhoeven for insights about Bug Planet.

\section{REFERENCES}

Bressler, S. and Grantham, C. E. (2000). Communities of Commerce: Building Internet Business Communities to Accelerate Growth, Minimize Risk, and Increase Customer Loyalty. McGraw-Hill.

Carotenuto, L., Etienne, W., Fontaine, M., Friedman, J., Newberg, H., Muller, M., Simpson, M., Slusher, J., and Stevenson, K. (1999). CommunitySpace: Toward flexible support for voluntary knowledge communities. In Proceedings of the Workshop "Changing Places", London, UK.

Colony, G.F. (2000). My View: Empowered Fruit Flies. Forrester Research, June, p2.

Grudin, J. (2001). Desituating action: Digital representation of context. Human-Computer Interaction, 16(2-3).

Ebbinghouse, C. (2001). You have been misinformed-now what?: Attacking dangerous data. Searcher, 9(4).

Kalish, J. (1997). P.R. firms surf the net. Reuters. Available online at http://www.mcspotlight.org/media/press/reuters_14feb97.html.

Lueg, C. (2000). Supporting social navigation in Usenet newsgroups. In Proceedings of the Workshop "Social Navigation-A Design Approach?" at the Annual ACM SIGCHI Conference on Computer-Human Interaction (CHI 2000), The Hague, The Netherlands.

Lueg, C. (2001a). Virtual Communities as Challenges to Real Companies. In Proceedings of the Pacific Asia Conference on Information Systems (PACIS 2001), Seoul, Korea

Lueg, C. (2001b). The Role of Information Systems in Information-Level Security Management. Submitted to ACIS 2001.

Manktelow, N. (2001). Net chatter is a data goldmine. IT News from The Age and the Sydney Morning Herald http://www.it.fairfax.com.au/e-commerce/20010227/A254532001Feb27.html. 
Reuters (1997). P.R. Firms Seek Image Control on the Internet. http://www.infowar.com/class_1/class1_zn.html-ssi.

Roberts, T. L. (1998). Are newsgroups virtual communities? In Proceedings of the Annual ACM SIGCHI Conference on Human Factors in Computing Systems (CHI'98), pages 360367, New York, NY. ACM Press.

Ulfelder, S. (1997). Lies, damn lies and the Internet. Computerworld. http://www.computerworld.com/cwi/story/0,1199,NAV47STO6800,00.htm.

Wellman, B. and Gulia, M. (1999). Virtual communities as communities. In Smith, M. A. and Kollock, P., editors, Communities in Cyberspace, pages 167-194. Routledge, London, UK. Williams, R. L. and Cothrel, J. (2000). Four smart ways to run online communities. Sloan Management Review, 41(4):81-91. 